\title{
Anisotropic optical properties of single-crystal $\mathrm{GdBa}_{2} \mathrm{Cu}_{3} \mathrm{O}_{7-\delta}$
}

\author{
V. Železny ${ }^{1}$, D.B. Tanner ${ }^{1}$, K. Kamarás ${ }^{2}$, L.P. Kozeeva ${ }^{3}$, A.A. Pavlyuk ${ }^{3}$ \\ ${ }^{1}$ Department of Physics, University of Florida, Gainesville, FL 32611, USA \\ ${ }^{2}$ Research Institute for Solid State Physics of the Hungarian Academy of Sciences, Budapest, Hungary \\ ${ }^{3}$ Institute of Inorganic Chemistry, Lavrenteva 3, 630090 Novosibirsk, Russia
}

Received: 20 June 1994

\begin{abstract}
The optical spectrum of reduced- $T_{c}$ $\mathrm{GdBa}_{2} \mathrm{Cu}_{3} \mathrm{O}_{7-\delta}$ has been measured for polarizations parallel and perpendicular to the $a b$ plane. The sample was an oxygen-deficient single crystal with a large face containing the $c$ axis. The polarized reflectance from this face was measured from $20-300 \mathrm{~K}$ in the spectral region from $30-3000 \mathrm{~cm}^{-1}$, with $300 \mathrm{~K}$ data to $30000 \mathrm{~cm}^{-1}$. Kramers-Kronig analysis was used to determine the spectral dependence of the $a b$ and the $c$ components of the dielectric tensor. The optical properties are strongly anisotropic. The $a b$-plane response resembles that of other reduced- $T_{c}$ materials whereas the $c$ axis, in contrast, shows only the presence of several phonons. There is a complete absence of charge carrier response along $c$ above and below $T_{c}$. This observation allows us to set an upper limit to the free-carrier spectral weight for transport perpendicular to the $\mathrm{CuO}_{2}$ planes.
\end{abstract}

PACS: 74.30.Gn; 74.70.Vy; 78.30.Er; 78.65.Ez

\section{Introduction}

The amount of anisotropy between the highly-conducting $a b$ planes and the perpendicular $c$ axis in the oxide superconductors is important because it is related to the effective dimensionality of these materials. In turn, the dimensionality has implications for the theoretical approach that describes the electronic structure. For example, if the high $T_{c}$ superconductors are basically anisotropic three-dimensional solids, then one can imagine them to have a Fermi surface and may take a Fermiliquid approach towards their properties. In this context, we note that band-structure calculations [1] give metallic behavior in all three directions. If, in contrast, they are highly two dimensional, then the Fermi-liquid approach may not be appropriate. Conventional electronic theory

* Permanent address: Institute of Physics, CSAV, Prague, Czechoslovakia does not permit a system to be metallic in two directions and insulating (or localized) in a third [2,3].

Electrical transport measurements find that the $c$-axis has a much smaller conductivity than the $a b$ plane [4]. In a few samples, notably fully oxygenated $\mathrm{YBa}_{2} \mathrm{Cu}_{3} \mathrm{O}_{7-\delta}$, the electrical resistance appears to be rather metallic $\left(d \rho_{c} / d T>0\right)$ along $c$, suggesting anisotropic three-dimensional behavior $[5,6]$. However other $\mathrm{YBa}_{2} \mathrm{Cu}_{3} \mathrm{O}_{7-\delta}$ samples and other oxide superconductors are more two dimensional, with resistance metallic in the $a b$ plane and insulating along $c$ [7]. Indeed, Forro et al. [8] argue on the basis of pressure studies that the $c$-axis transport is diffusive even in samples for which $d \rho_{c} / d T$ is positive.

Polarized optical spectroscopy can provide important information about the dimensionality, estimating not only the conductivity but also the oscillator strength or spectral weight for transitions along principal directions. These measurements are difficult for the $c$ axis, because crystals of this family have the habit to grow in thin plates with minimum dimension along $c$ while thin films tend to grow with the $c$ axis normal to the substrate surfaces. Nevertheless, there have been several $c$-axis polarized measurements reported. Room temperature measurements on $\mathrm{La}_{2-x} \mathrm{Sr}_{x} \mathrm{CuO}_{4}$ samples which were metallic [9] and even superconducting [10] and on metallic $\mathrm{La}_{2} \mathrm{NiO}_{4}$ samples [11] find essentially insulating behavior along $c$. In this context, "insulating behavior" means the absence of electronic aborption in the far-infrared region, so that the spectrum is dominated by features associated with optical phonons. Tamasaku et al. [10] showed that below $T_{c}$ superconducting samples of $\mathrm{La}_{2-x} \mathrm{Sr}_{x} \mathrm{CuO}_{4}$ develop a sharp plasma edge - a measure of the superfluid density. Recent measurements of $\mathrm{Bi}_{2} \mathrm{Sr}_{2} \mathrm{CaCu}_{2} \mathrm{O}_{8}$ find highly insulating $c$-axis reflectance, both above and below $T_{c}[12]$.

$\mathrm{YBa}_{2} \mathrm{Cu}_{3} \mathrm{O}_{7-\delta}$ is an important material for the study of the $c$ axis, on account of the variation in $c$ axis transport that can be obtained, either by doping or by variation in the oxygen content $\delta$. Several studies have reported metallic reflectance for $c$-axis polarization in $\mathrm{YBa}_{2} \mathrm{Cu}_{3} \mathrm{O}_{7-\delta}$. Bozovic et al. [13] reported a stronglydamped plasma edge near $0.1 \mathrm{eV}$ from reflectance mea- 
surements of $\mathrm{YBa}_{2} \mathrm{Cu}_{3} \mathrm{O}_{7-\delta}$ crystals using an infrared microscope. Kamba et al. [14] reported $c$-polarized spectra for films grown on (110) $\mathrm{SrTiO}_{3}$ substrates; however, this experiment was not completely successful on account of imperfect orientation of the $c$ axis. There are other papers reporting on anisotropy in the near ir-visible-near uv regions [15-19].

In the far infrared region, Collins et al. [20] claimed to measure the anisotropy of the superconducting gap on a mosaic of about 12 crystals with polished surfaces. Perhaps the first reliable single crystal data were presented by Koch et al. [21]. Above $T_{c}$ an overdamped plasma edge seems to appear in the $c$-axis spectrum while below $T_{c}$ a very sharp plasma-like reflectance edge occurs at $100 \mathrm{~cm}^{-1}$. Homes et al. [22] have measured reduced $T_{c}$ crystals of $\mathrm{YBa}_{2} \mathrm{Cu}_{3} \mathrm{O}_{7-\delta}\left(T_{c} \approx 63 \mathrm{~K}\right)$. $c$-axis polarized electronic absorption is seen in the far infrared for both the normal and superconducting states. Below $T_{c}$, there is a dramatic sharpening of a plasma-like edge at about $60 \mathrm{~cm}^{-1}$ in the reflectance and evidence for a pseudogap at about $200 \mathrm{~cm}^{-1}$ in the optical conductivity. Above $T_{c}$ the plasma edge is overdamped and the pseudogap absent.

The effect of oxygen variation on the $c$-axis spectra of $\mathrm{YBa}_{2} \mathrm{Cu}_{3} \mathrm{O}_{7-\delta}$ has been measured by Dürrler et al. [23] and Cooper et al. [24,25]. The data were taken at room temperature. Both measurements show a strongly damped electronic band for $\delta \approx 0$ whose strength decreased with increasing $\delta$. One thing to note is that at all values of $\delta$ the $c$-axis spectra clearly show optical phonon features, so the presence of phonon signatures are not by themselves evidence for "insulating" behavior; instead, one looks for small (or zero) optical conductivity at frequencies between the phonon features or for near zero reflectance at the longitudinal-optical phonon minima in the reflectance. (The latter indicates a small value of $\sigma_{1}(\omega)$ at the longitudinal optical frequency.) By these criteria, only the tetragonal $\mathrm{YBa}_{2} \mathrm{Cu}_{3} \mathrm{O}_{6}$ spectra of Dürrler et al. [23] can be said to be highly insulating.

In this paper we report the anisotropy of the far-infrared reflectance parallel to and perpendicular to the $a b$ plane of $\mathrm{GdBa}_{2} \mathrm{Cu}_{3} \mathrm{O}_{7-\delta}$. Our samples are deficient in oxygen content and inadvertently doped with $\mathrm{Al}$, so that the transition temperature is $\sim 30 \mathrm{~K}$. This value puts it at the low end of the range where superconductivity occurs in the $R \mathrm{Ba}_{2} \mathrm{Cu}_{3} \mathrm{O}_{7-\delta}$ system. The crystal studied in detail was unusually large, $5 \times 4 \times 1.5 \mathrm{~mm}^{3}$, and had a facet with excellent optical quality containing the $c$ axis, allowing polarized reflectance measurements to be made without recourse to mosaics, polishing, or microscopes with their steeply converging beams.

$\mathrm{GdBa}_{2} \mathrm{Cu}_{3} \mathrm{O}_{7-\delta}$ is isomorphic with $\mathrm{YBa}_{2} \mathrm{Cu}_{3} \mathrm{O}_{7-\delta}$; the only difference is the substitution of $\mathrm{Gd}$ for $\mathrm{Y}$. As is well known, this substitution has minimal effect on the physical properties, including superconductivity, with the exception that the unpaired electrons of the $\mathrm{Gd}^{3+}$ ions give a strong paramagnetic susceptibility and undergo a phase transition at $2.2 \mathrm{~K}$ into an antiferromagnetic state.

\section{Experimental details}

The $\mathrm{GdBa}_{2} \mathrm{Cu}_{3} \mathrm{O}_{7-\delta}$ crystals were grown by spontaneous crystallization from a high-temperature melt consisting of $0.7 \% \mathrm{Gd}_{2} \mathrm{O}_{3}, 29 \% \mathrm{BaO}$, and $70 \% \mathrm{CuO}$. This $\mathrm{Cu}$-rich and Gd-poor mixture was heated in an alumina crucible to $1060 \mathrm{C}$ and held there for $5 \mathrm{~h}$. The system was then cooled at a rate of 10 degrees/day to $940 \mathrm{C}$. The remaining liquid was decanted while still in the oven, to avoid thermal stress on the crystals. After that, the crystals were cooled to room temperature at a rate of 10-20 degrees $/ \mathrm{h}$. Crystals obtained by this method ranged in size up to a maximum of $5 \times 4 \times 1.5 \mathrm{~mm}^{3}$. Elemental analysis showed that the crystals had acquired a considerable amount of $\mathrm{Al}$ from the cruicible; the composition was found to be $\mathrm{GdBa}_{1.8} \mathrm{Cu}_{2.5} \mathrm{Al}_{0.25} \mathrm{O}_{7-\delta}$. Further details of the growth of these crystals will be presented elsewhere [26].

Several techniques were used to characterize our samples. The dc electrical resistance shows two superconducting drops: one partial at $90 \mathrm{~K}$ and the second to zero resistance at $30 \mathrm{~K}$. The magnitude of the resistance is quite high in comparison to high quality samples. The optical measurements, discussed below, suggest that the room-temperature resistivity is $\sim 700 \mu \Omega-\mathrm{cm}$, compared to $\sim 200 \mu \Omega-\mathrm{cm}$ for fully oxygenated crystals or films of $\mathrm{YBa}_{2} \mathrm{Cu}_{3} \mathrm{O}_{7}$.

The magnetic susceptibility, which we also measured, shows a small deviation from a paramagnetic $1 / T$ dependence at $87 \mathrm{~K}$. The oxygen content $\delta$ was estimated from the Raman spectrum $[27,28]$. From this we concluded that the best description of our crystals would be by the formula $\mathrm{GdBa}_{2} \mathrm{Cu}_{3} \mathrm{O}_{6.4}$. This value means that in the phase diagram they are located in the vicinity of the metal/insulator transition but on the metallic side. (The position of this transition is now considered to be at $\mathrm{O}_{6.35}$ $[29,30]$.) The estimated oxygen content is consistent with the lower transition in the resistivity, so we take $T_{c}$ to be $30 \mathrm{~K}$. Observation of the crystal surfaces under a polarizing microscope revealed $a b$ microtwinning, which prevented us from distinguishing anisotropy in the $a b$ plane. This observation, however, demonstrates that the sample had the orthorhombic crystal structure necessary for superconductivity in the $R \mathrm{Ba}_{2} \mathrm{Cu}_{3} \mathrm{O}_{7-\delta}$ system.

We made reflectance measurements on two faces of the crystal, one which contained the $a b$ plane and one which contained the $c$ axis, using a Bruker IFS $113 \mathrm{v}$ interferometric spectrometer over $50-5000 \mathrm{~cm}^{-1}$ $(0.006-0.6 \mathrm{eV})$ and using Perkin-Elmer $16 \mathrm{U}$ spectrometer over $1000-30000 \mathrm{~cm}^{-1}(0.12-3.7 \mathrm{eV})$. For our measurements we used the natural crystal surface unmodified by polishing or any other treatment. The reflectance was measured relative to an $\mathrm{Al}$ reference mirror and corrected for the known reflectance of Al. After measurements, the surface of our crystal was coated with $\mathrm{Al}$ and the reflectance of the coated sample measured, in order to determine accurately the sample area and to estimate the diffuse scattering due to any imperfections in the surface. A continuous-flow cryostat was used to cool the sample to a base temperature of $20 \mathrm{~K}$. 


\section{Results}

The reflectance from the facet containing the $c$-axis is completely different for the two polarizations, as shown in Figs. 1-3. Figure 1 shows the $300 \mathrm{~K}$ reflectance over $100-30000 \mathrm{~cm}^{-1}(0.012-3.7 \mathrm{eV})$ for both the $a b$-plane and the $c$-axis directions. The $a b$-plane reflectance shows a rapid decrease with increasing frequency. There is a weak structure around $5000 \mathrm{~cm}^{-1}(0.6 \mathrm{eV})$ and an-

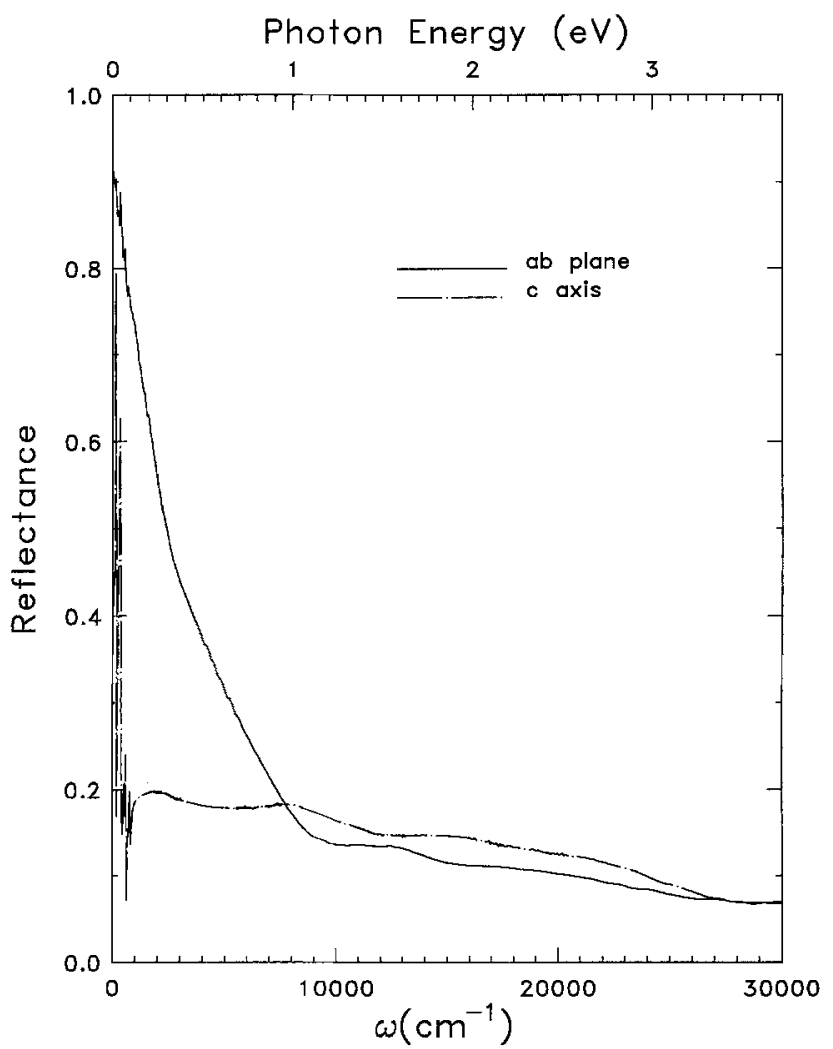

Fig. 1. Polarized reflectance of $\mathrm{GdBa}_{2} \mathrm{Cu}_{3} \mathrm{O}_{7-\delta}$ at room temperature

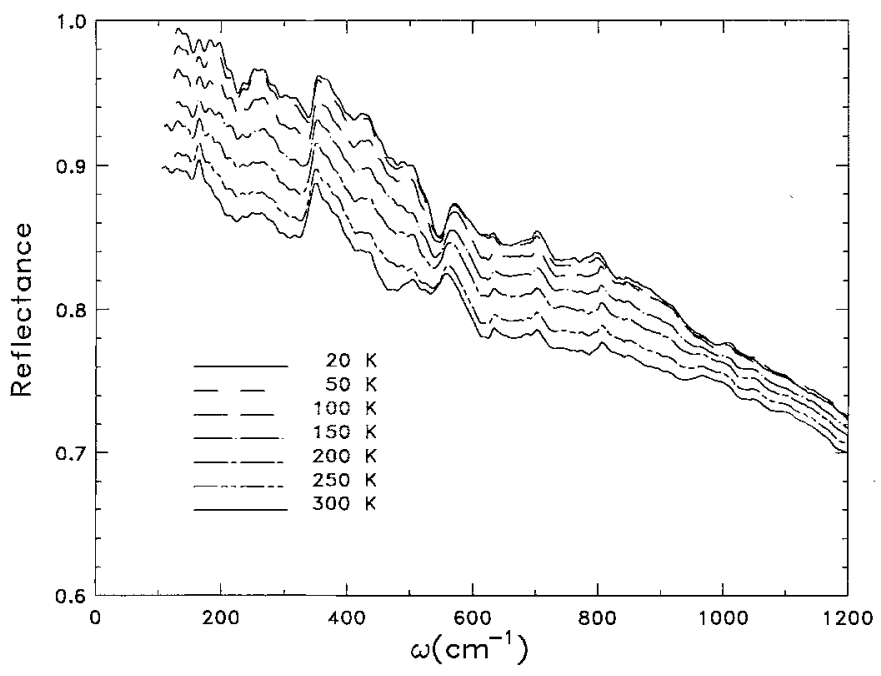

Fig. 2. The reflectance of $\mathrm{GdBa}_{2} \mathrm{Cu}_{3} \mathrm{O}_{7-\delta}$ for radiation polarized along the $a b$ plane

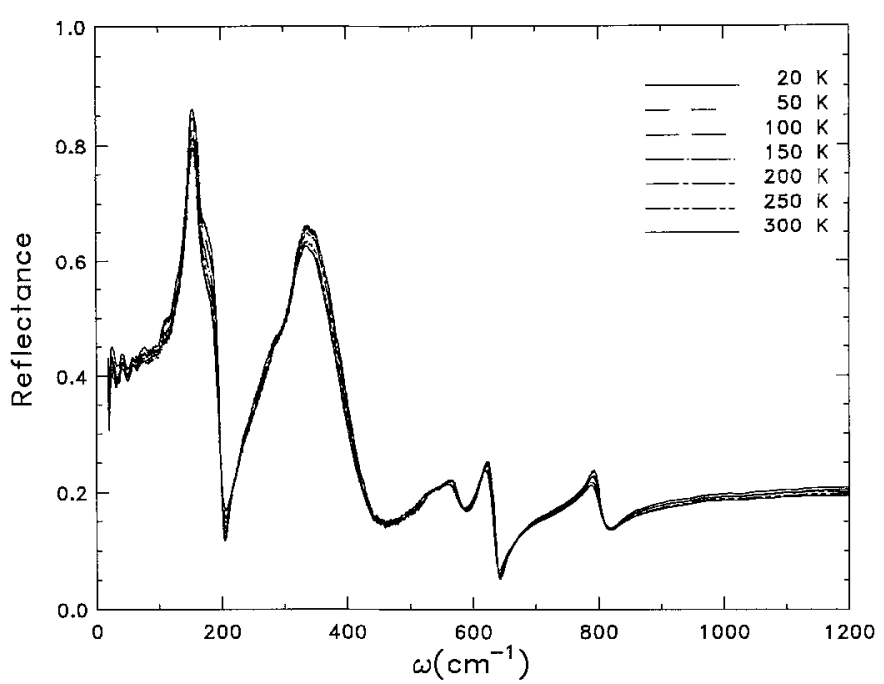

Fig. 3. The reflectance of $\mathrm{GdBa}_{2} \mathrm{Cu}_{3} \mathrm{O}_{7-\delta}$ for radiation polarized along the $c$ axis

other around $12000 \mathrm{~cm}^{-1}(1.5 \mathrm{eV})$. The data, including these weak features, resemble closely the $T_{c}=30 \mathrm{~K}$ $\mathrm{YBa}_{2} \mathrm{Cu}_{3} \mathrm{O}_{7-\delta}$ sample reported by Orenstein et al. [31]. The $c$-axis spectrum is quite flat. The low frequency structure is associated with optical phonons. In addition, there are broad bands around $8000 \mathrm{~cm}^{-1}$ and $16000 \mathrm{~cm}^{-1}$ ( 1 and $2 \mathrm{eV}$, respectively) and a minimum at our upper frequency limit. The data differ strongly from the $c$-axis reflectance of the tetragonal phase $(\delta \geqslant 0.8)$, which is dominated by a strong feature at $3.8 \mathrm{eV}$ [19]. In particular, Kircher et al. [19] show that there is a rising reflectance over $2-3.8 \mathrm{eV}$ in the highly oxygen deficient material whereas samples with small values of $\delta$ have a decreasing reflectance in this range. These comparisons give us confidence in our characterization of the sample as reduced $T_{c}$, superconducting $\mathrm{GdBa}_{2} \mathrm{Cu}_{3} \mathrm{O}_{7-\delta}$.

Figure 2 shows the $a b$-plane-polarized reflectance at temperatures between 20 and $300 \mathrm{~K}$. These spectra are similar to those reported in many previous measurements [31-33]. At the lowest temperatures, the reflectance is close to unity below $150 \mathrm{~cm}^{-1}$. However, the characteristic shoulder or knee around $430 \mathrm{~cm}^{-1}$ that is commonly seen in the $\mathbf{E} \| a b$ spectrum does not occur in the spectra of Fig. 2. Weak features at 150,350 , and $560 \mathrm{~cm}^{-1}$ are due to phonons, visible on account of the reduced carrier concentration in this sample. As temperature is increased, the reflectance drops, with the decrease largest at the lowest frequencies.

In contrast to the electronic absorption which dominates the $a b$-plane optical properties, the reststrahlen bands of optical phonons are the principal features of the $c$-axis reflectance, shown in Fig. 3. This reflectance is characteristic of a semiconductor or insulator, with quite deep minima at the longitudinal optical frequencies. As $\omega \rightarrow 0$, the reflectance decreases and approaches a constant value $\mathscr{R}=0.4$. There is insignificant contribution of free carriers to the spectrum. There is also no clear evidence for appearance of the midinfrared absorption well-known from $a b$-plane measurements. The tempera- 
ture dependence of the spectra is practically negligible; there is only a small amount of sharpening of the phonons at low temperatures. In particular, the spectra are the same above and below the temperature where the material is superconducting. The onset of superconductivity has no effect on the c-axis spectrum in the $30-3000 \mathrm{~cm}^{-1}$ range.

\section{Kramers-Kronig analysis}

\section{A. ab-plane results}

We used Kramers-Kronig analysis to determine the optical conductivity, $\sigma_{1}(\omega)$, and the real part of the dielectric function, $\varepsilon_{1}(\omega)$, from our reflectance data. We used different low-frequency extrapolations for the two polarizations: for $\mathbf{E} \| a b$ we used a Hagen-Rubens formula $\mathscr{R}=1-A \sqrt{\omega}$ above $T_{c}$. On account of the $\sim 100 \%$ reflectance, below $T_{c}$, the reflectance was extrapolated as $\mathscr{R}=1$ in this temperature range. For $\mathbf{E} \| c$ we used a constant reflectance below the lowest measured frequency [34]. At high frequencies a power law continuation was used.

The $a b$-plane dielectric function, shown in Fig. 4, is negative at low frequencies, as expected for a conductor, with a zero-crossing at $3700 \mathrm{~cm}^{-1}$ in the near infrared. At low temperatures, $\varepsilon_{1}(\omega)$ is well described by $\varepsilon_{1}(\omega)=\varepsilon_{\infty}-\omega_{p}^{2} / \omega^{2}$, as has been shown previously [34]. For our sample, we find $\omega_{p} \approx 5700 \mathrm{~cm}^{-1}$. A derivativelike structure from the $a b$-plane optical phonons can also be seen superimposed on the electronic background.

The $a b$-plane optical conductivity, shown in Fig. 5, is similar to those published earlier for superconducting samples. Above $T_{c}$ there is a low-frequency upturn due to the response of the charge carriers, which is Drudelike at low frequencies and which has a non-Drude falloff in the midinfrared range. With decreasing temperature, the zero-frequency conductivity increases and the width of the low frequency upturn narrows until $T_{c}$ is reached. Below $T_{c}$, the low-frequency conductivity is

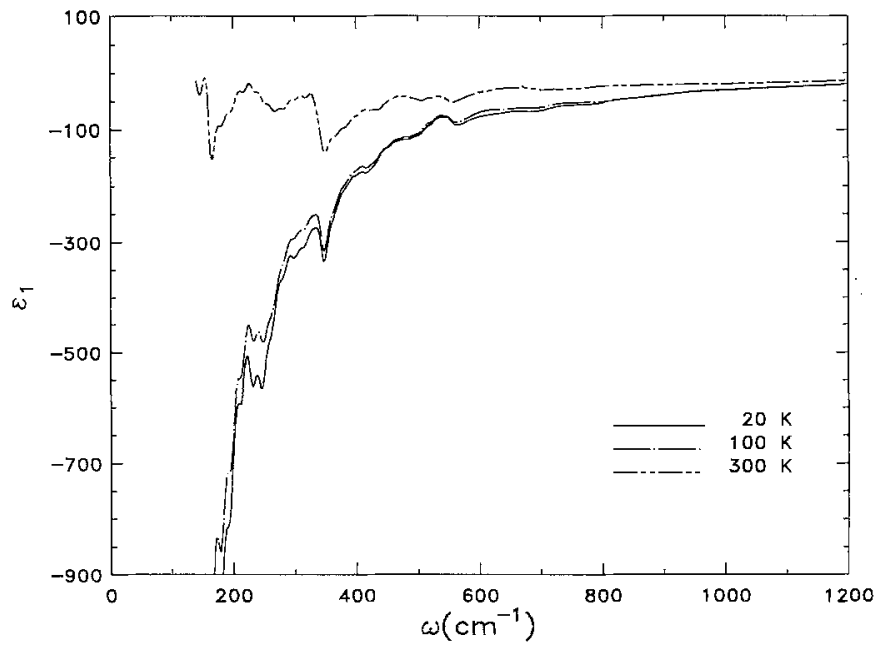

Fig. 4. The $a b$-plane dielectric function $\varepsilon_{1}(\omega)$ of a crystal of $\mathrm{GdBa}_{2} \mathrm{Cu}_{3} \mathrm{O}_{7-\delta}$

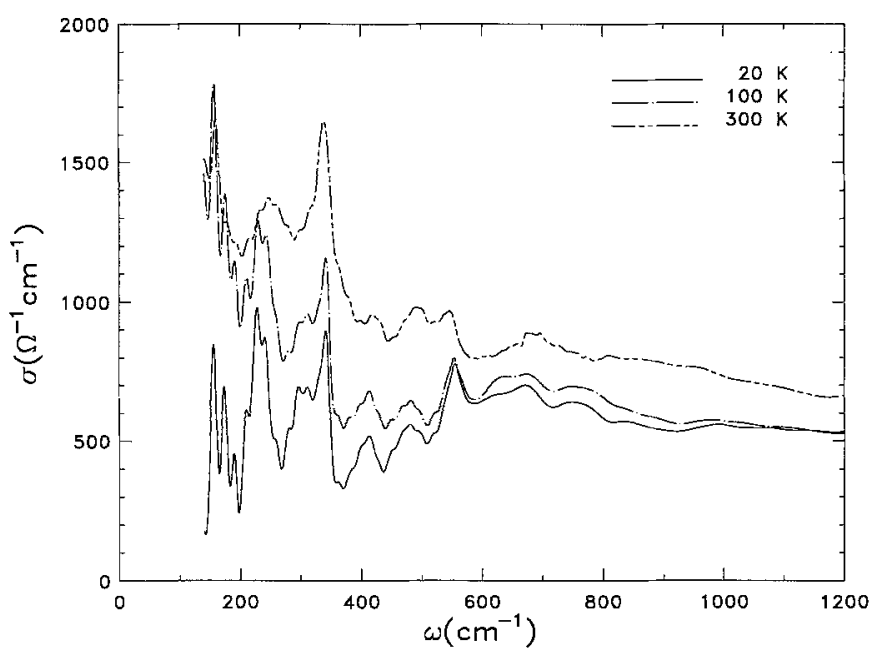

Fig. 5. The $a b$-plane optical conductivity $\sigma_{1}(\omega)$ of a crystal of $\mathrm{GdBa}_{2} \mathrm{Cu}_{3} \mathrm{O}_{7-\delta}$

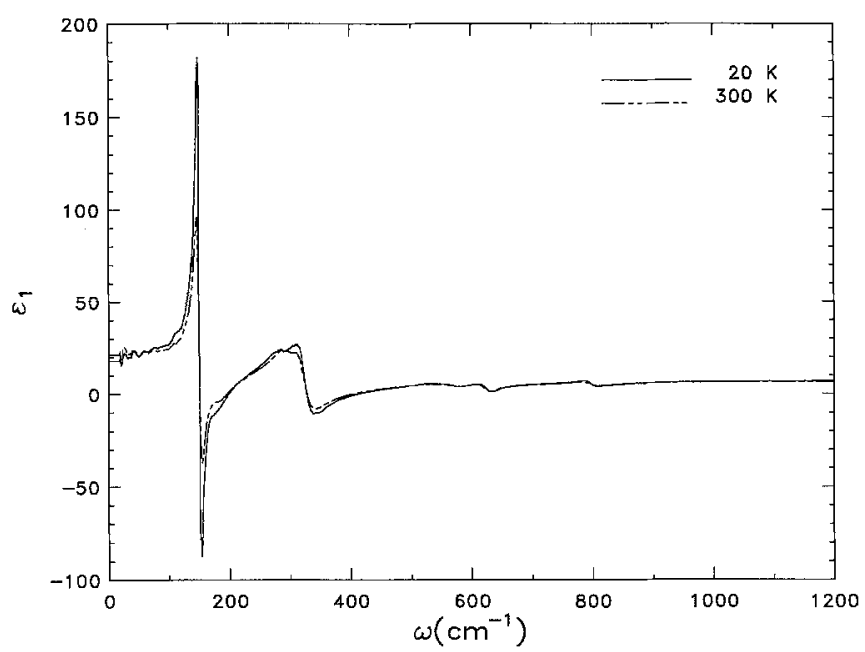

Fig. 6. The dielectric function $\varepsilon_{1}(\omega)$ for $\mathbf{E} \| c$ of a crystal of $\mathrm{GdBa}_{2} \mathrm{Cu}_{3} \mathrm{O}_{7-\delta}$

sharply reduced as the charge carriers condense into the superfluid. Several sharp $a b$-plane phonon modes can be seen superimposed on the electronic background of Fig. 5 . The frequencies of these phonons are listed in Table 1 .

\section{B. $c$-axis results}

For $\mathbf{E} \| c$, the real dielectric function, shown in Fig. 6 for our highest and lowest measurement temperatures, is positive everywhere except in the phonon reststrahlen bands, between transverse and longitudinal phonon frequencies. The low-frequency limiting value is $\varepsilon_{1}(0)=21$ while the midinfrared value is $\varepsilon_{\text {opt }}=7$. Most of the oscillator strength is in the lowest band at $148 \mathrm{~cm}^{-1}$. The values for $\varepsilon_{1}(0)$ and $\varepsilon_{\text {opt }}$ are in good agreement with those for other perovskites when the contributions of soft modes are subtracted.

The phonons are essentially the only things that can be seen in the $c$-axis-polarized optical conductivity, which 


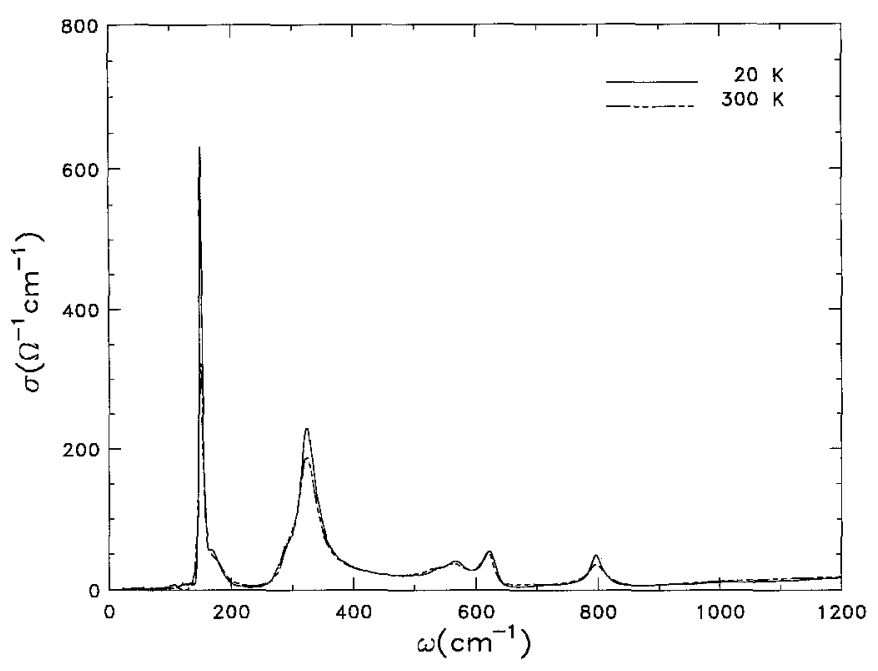

Fig. 7. The optical conductivity $\sigma_{1}(\omega)$ for $\mathbf{E} \| c$ of a crystal of $\mathrm{GdBa}_{2} \mathrm{Cu}_{3} \mathrm{O}_{7-\delta}$

Table 1. Frequencies (in $\mathrm{cm}^{-1}$ ) of Phonon features in $\mathrm{GdBa}_{2} \mathrm{Cu}_{3} \mathrm{O}_{7-\delta}$

\begin{tabular}{llllllllll}
\hline Polarization & & & & & & & & \\
\hline$a b$ plane & 150 & & 227 & 333 & 420 & 493 & 547 & 680 \\
$c$ axis & 148 & 176 & 288 & 320 & & & 560 & 620 \\
\hline
\end{tabular}

Note: An additional $c$-axis-polarized band is seen at $800 \mathrm{~cm}^{-1}$, above the maximum of the one-phonon frequencies

is shown in Fig. 7. The data are from $20 \mathrm{~K}$ and $300 \mathrm{~K}$ reflectance measurements; there is only small temperature dependence in this polarization. The phonon frequencies are listed in Table 1. These phonons are grouped into three doublets (some are seen as shoulders) and one singlet. The frequencies are in good agreement with reports in the literature for the $c$-axis phonons $[20,21]$ in $\mathrm{YBa}_{2} \mathrm{Cu}_{3} \mathrm{O}_{7}$ and definitely different from those for the $c$ axis phonons [35] in $\mathrm{YBa}_{2} \mathrm{Cu}_{3} \mathrm{O}_{6}$. This result is further confirmation that our sample is representative of superconducting material.

The spectrum also has a clear conductivity peak at $800 \mathrm{~cm}^{-1}$. This frequency is well above the highest phonon frequency in neutron density of states measurements [36] and therefore cannot be assigned to a single-phonon absorption. We suggest it is due to a two-phonon process related to the known anomalies [37] in the phonon spectrum around $400 \mathrm{~cm}^{-1}(50 \mathrm{meV})$, although a magnetic absorption cannot be ruled out.

\section{Discussion}

\section{A. ab-plane spectra}

The characteristic notch in the conductivity, well known from $a b$-plane measurements [31-34], is very difficult to see in Fig. 5. One reason is the presence of phonon structure in this spectral region. However, there is a direct relation between the absence of the notch in the conductivity spectrum and the missing knee in the reflectance spectrum of Fig. 2. The difference between these spectra and other $a b$-plane spectra may well be explained by the effect discovered by Reedyk et al. [38]. The spectra differ due to the direction of incidence of the light: along $c$ in most measurements but perpendicular to $c$ in the one reported here. This effect has been studied in some detail by Reedyk et al. [38] in $\mathrm{Pb}_{2} \mathrm{Sr}_{2}(\mathrm{Y} / \mathrm{Ca}) \mathrm{Cu}_{3} \mathrm{O}_{9-\delta}$.

\section{B. c-axis spectra}

Comparing our data to previous measurements of the $c$ axis, we find some similarity with the measurements of Koch et al. [21] and Homes et al. [22]. These two measurements find nearly the same positions of the phonon bands as occur in our spectra. We find less agreement with the spectra of Collins et al. [20]. In the latter case, the data look more like the unpolarized spectra of ceramic samples, perhaps on account of the mosaic of several small crystals used.

The key difference between the spectra presented here and those of other groups [20-22,24,25] is the absence of any contribution of free carriers to our $c$-axis conductivity. This difference must be due to the reduced $T_{c}$ of our crystals. The other samples that have been studied had $T_{c}$ near $90 \mathrm{~K}[20,21]$ and $63 \mathrm{~K}[22]$. In our case, the doping level was considerably lower $(\delta \approx 0.6)$ and $T_{c}=30 \mathrm{~K}$.

The absence of any free carrier contribution to our $c$ polarized spectra has interesting consequences. From a two-component analysis of the $a b$-plane spectra, we find that most of the free carriers condense into the superfluid, with plasma frequency $\omega_{\mathrm{ps}_{a b}}=5700 \mathrm{~cm}^{-1}$. This leads to an estimate of the $a b$-plane penetration depth of $\lambda_{a b}=2800 \AA$. In contrast, the screened plasma frequency for $c, \widetilde{\omega}_{\mathrm{ps}_{c}}=\omega_{\mathrm{ps}_{c}} / \sqrt{\varepsilon}$ must be at a frequency lower than $50 \mathrm{~cm}^{-1}$. Otherwise, we would see a plasma minimum similar to what was reported by Tamasaku et al. [10] for $\mathrm{La}_{2-x} \mathrm{Sr}_{x} \mathrm{CuO}_{4}$. Taking $\varepsilon=21$ we get $\omega_{\mathrm{ps}_{a b}} \leqq 230 \mathrm{~cm}^{-1}$ or a penetration depth of $\lambda_{c} \geqq 7 \mu$.

Assuming a one-electron band scheme and using a three-dimensional tight-binding model, $\omega_{p_{c}}$ is given by

$$
\begin{aligned}
\omega_{p_{c}}^{2} & =\frac{e^{2}}{\hbar^{2} \varepsilon_{0}} \sum_{\text {В.Z. }} \frac{\partial^{2} \varepsilon_{k}}{\partial k_{\gamma}^{2}} f\left(\varepsilon_{k}\right) \\
& =\frac{4 t_{c} c^{2} e^{2}}{\pi \varepsilon_{0} \hbar^{2} V} \sin \left(\frac{\pi \rho}{2}\right)
\end{aligned}
$$

where $f\left(\varepsilon_{k}\right)$ is the Fermi-Dirac occupation function, $t_{c}$ is the transfer integral, $c$ is the lattice constant along the $c$ axis, $V$ is the unit cell volume, $\varepsilon_{0}$ is the dielectric constant of vacuum, and $\rho$ is the site occupancy. Equation (1) gives $t_{c} \leqq 0.045 \mathrm{meV}$. This value is extremely low, corresponding to a temperature of $0.6 \mathrm{~K}$. A three-dimensional model is therefore almost certainly not correct, because with this small a value for $t_{c}$, the Fermi surface would be open in the direction of the $c$ axis. With an open Fermi surface, only a certain portion of the charge carriers can contribute to intraband optical transitions polarized $E \| c$, so that $t_{c}$ is in fact larger than inferred 
above. This effect was first pointed out by Kwak [39] in the context of organic conductors. Using

$\frac{\omega_{p_{c}}}{\omega_{p_{a b}}}=\sqrt{2} \frac{c t_{c}}{a E}$,

where $a$ is the lattice constant along the $a$ axis and $E$, defined by

$\omega_{p_{a b}}^{2}=\frac{2 e^{2} a^{2} E}{\hbar^{2} V}$

is a function of band width and band filling. This analysis gives $t_{c} \leqq 0.75 \mathrm{meV}$, corresponding to a temperature of $9 \mathrm{~K}$. Thus, down to $\sim 9 \mathrm{~K}$ there is no correlation of electron motion along the $c$ axis. Note that this temperature is definitely below the superconducting transition temperature.

Our results show that the electronic properties of oxygen deficient $\mathrm{GdBa}_{2} \mathrm{Cu}_{3} \mathrm{O}_{7-\delta}$ are strongly anisotropic. It behaves like a metal, albeit an unusual metal, in the $a b$ plane while along the $c$ axis it looks like an insulator. Because our sample is definitely superconducting, this result has clear implications for the electronic structure of the oxide superconductors. Superconductivity can occur in samples which have no coherent transport along $c$. Note, however, that unlike expectations for two-dimensional models [40], - and in contrast with the results for $\mathrm{La}_{2-x} \mathrm{Sr}_{x} \mathrm{CuO}_{4}$ [10] - our sample shows no development of the plasma edge of the superfluid below $T_{c}$. Thus, even well below $T_{c}$, there is a strongly two-dimensional flavor to its optical properties.

We would like to acknowledge enthusiastic discussions with P.W. Anderson, P.B. Littlewood, J.R. Schrieffer, and T. Timusk. Work at Florida is supported by NSF grants DMR-9101676 and DMR-9403984. Research in Hungary supported by the Hungarian National Foundation for Scientific Research under Grant No. 2950 and by the Hungarian-American Joint Fund grant $92 b-271$.

\section{References}

1. Pickett, W.E.: Rev. Mod. Phys. 61, 433 (1989)

2. Anderson, P.W.: Int. J. Mod. Phys. B4, 181 (1990)

3. Anderson, P.W.: Science 256, 1526 (1992)

4. For a review; see Cooper, S.L., Gray, K.E.: In: Physical properties of high temperature superconductors III, p. 61. Ginsberg, D.M. (ed.). Singapore: World Scientific 1994

5. Iye, Y., Tamegai, T., Sakakibara, T., Goto, T., Miura, N., Takeya, H., Takei, H.: Physica C153-155, 26 (1988)

6. Friedmann, T.A., Rabin, W.M., Giapintzakis, J., Rice, J.P., Ginsberg, D.M.: Phys. Rev. B42, 6217 (1990)

7. Hagen, S., Jing, T.W., Wang, Z.Z., Horvath, J., Ong, N.P.: Phys. Rev. B37, 7298 (1988)

8. Forro, L., Ilakovac, V., Cooper, J.R., Ayache, C., Henry, J.-Y.: Phys. Rev. B46, 6626 (1992)

9. Collins, R.T., Schlesinger, Z., Chandrashekhar, G.V., Shafer, M.W.: Phys. Rev. B39, 2251 (1989)

10. Tamasaku, K., Nakamura, Y., Uchida, S.: Phys. Rev. Lett. 69, 1455 (1992)

11. Bassat, J., Odier, P., Gervais, F.: Phys. Rev. B35, 7126 (1987)

12. Tajima, S., Gu, G.D., Miyamoto, S., Odagawa, A., Koshizuka, N.: Phys. Rev. B48, 16164 (1993)

13. Bozovic, I., Char, K., Yoo, S.J.B., Kapitulnik, A., Beasley, M.R., Geballe, T.H., Wang, Z.Z., Hagen, S., Ong, N.P., Aspnes, D.E., Kelly, M.K.: Phys. Rev. B38, 5077 (1988)
14. Kamba, S., Petzelt, J., Železný, V., Pechen, E.V., Krasnosvobodtsev, S.I., Gorshunov, B.P.: Solid State Commun. 70, 547 (1989)

15. Petrov, M.P., Grachev, A.I., Krasin'kova, M.V., Nechitailov, A.A., Prokofiev, V.V., Poborchy, V.V., Shagin, S.I., Kartenko, N.F.: Solid State Commun. 67, 1197 (1988)

16. Maksimov, E.G., Rashkeev, S.N., Savrasov, S.Y., Uspenskii, Y.A.: Phys. Rev. Lett. 63, 1880 (1989)

17. Kircher, J., Alouani, M., Garriga, M., Murugaraj, P., Maier, J., Thompson, C., Cardona, M., Andersen, O.K., Jepsen, O.: Phys. Rev. B44, 217 (1991)

18. Geserich, H.P., Koch, B., Dürrler, M., Wolf, Th.: In: Electronic properties of high $T_{c}$ superconductors and related compounds. Kuzmany, H., Mehring, M., Fink, J. (eds.). Springer Series in Solid State Science, Vol.99, p. 265. Berlin, Heidelberg, New York: Springer 1990

19. Kircher, J., Kelly, M.K., Rashkeev, S., Alouani, M., Fuchs, D., Cardona, M.: Phys. Rev. B44, 217 (1991)

20. Collins, R.T., Schlesinger, Z., Holtzberg, F., Feild, C.: Phys. Rev. Lett. 63, 422 (1989)

21. Koch, B., Dürrler, M., Geserich, H.P., Wolf, Th., Roth, G., Zachmann, G.: In: Electronic properties of high- $T_{c}$ superconductors and related compounds. Kuzmany, H., Mehring, M., Fink, J. (eds.). Springer Series in Solid State Sciences, Vol. 99, p. 290. Berlin, Heidelberg, New York: Springer 1990

22. Homes, C.C., Timusk, T., Liang, R., Bonn, D.A., Hardy, W.N.: Phys. Rev. Lett. 71, 1645 (1993)

23. Dürrler, M., Zibold, A., Wider, K., Geserich, H.P., Wolf, Th., Roth, G., Bräuchle, G., Erb, A., Müller-Vogt, G., Kircher, J.: In: Electronic properties of high- $T_{c}$ superconductors. Kuzmany, H., Mehring, M., Fink, J. (eds.). Springer Series in Solid State Science, Vol. 113, p. 265. Berlin, Heidelberg, New York: Springer 1993

24. Cooper, S.L., Nyhus, P., Reznik, D., Klein, M.V., Lee, W.C., Ginsberg, D.M., Veal, B., Paulikas, A.P., Dabrowski, B.: Phys. Rev. Lett. 70, 1533 (1993)

25. Cooper, S.L., Reznik, D., Kotz, A., Karlow, M.A., Liu, R., Klein, M.V., Lee, W.C., Giapintzakis, J., Ginsberg, D.M., Veal, B., Paulikas, A.P.: Phys. Rev. B47, 8233 (1993)

26. Kozeeva, L.P., Pavlyuk, A.A.: (unpublished)

27. Burns, G., Dacol, F.H., Field, C., Holtzberg, F.: Solid State Commun. 77, 367 (1991)

28. Železný, V., Tanner, D.B., Park, C., Anderson, T.J., Kozeeva, L.P., Pavlyuk, A.A.: Phys. Status Solidi b 177, K37 (1993)

29. Jorgensen, J.D., Veal, B.W., Paulikas, A.P., Nowicki, L.J., Crabtree, G.W., Claus, H., Kwok, W.K.: Phys. Rev. B41, 1863 (1990)

30. Cava, R.J., Hewat, A.W., Hewat, E.A., Batlogg, B., Marezio, M., Rabe, K.M., Krajewski, J.J., Peck, Jr. W.F., Rupp, Jr. L.W.: Physica C165, 419 (1990)

31. Orenstein, J., Thomas, G.A., Millis, A.J., Cooper, S.L., Rapkine, D.H., Timusk, T., Schneemeyer, L.F., Waszczak, J.V.: Phys. Rev. B42, 6342 (1990)

32. Timusk, T., Tanner, D.B.: In: Physical properties of high temperature superconductors I, p. 339. Ginsberg, D.M. (ed.). Singapore: World Scientific 1989

33. Tanner, D.B., Timusk, T.: In: Physical properties of high temperature superconductors III, p. 363. Ginsberg, D.M. (ed.). Singapore: World Scientific 1992

34. Kamarás, K., Herr, S.L., Porter, C.D., Tache, N., Tanner, D.B., Etemad, S., Venkatesan, T., Chase, E., Inam, A., Wu, X.D., Hegde, M.S., Dutta, B.: Phys. Rev. Lett. 64, 84 (1990)

35. Crawford, M.K., Burns, G., Holtzber, F.: Solid State Commun. 70, 557 (1989)

36. Renker, B., Gompf, F., Gering, E., Ewert, D., Rietschek, H., Dianoux, A.: Z. Phys. B73, 309 (1988)

37. Reichard, W., Pyka, N., Pintschovius, L., Hennion, B., Collin, G.: Physica C162-164, 464 (1989)

38. Reedyk, M., Timusk, T.: Phys. Rev. Lett. 69, 2705 (1992)

39. Kwak, J.F.: Phys. Rev. B26, 4789 (1982)

40. Anderson, P.W.: Physica C185-189, 11 (1991) 